University of Nebraska - Lincoln

DigitalCommons@University of Nebraska - Lincoln

Faculty Publications in Food Science and Technology

2015

Promises and Prospects of Microbiome Studies

Maria C. Rivera

Jacques Izard

Follow this and additional works at: https://digitalcommons.unl.edu/foodsciefacpub

Part of the Food Science Commons, Genomics Commons, and the Other Genetics and Genomics Commons

This Article is brought to you for free and open access by the Food Science and Technology Department at DigitalCommons@University of Nebraska - Lincoln. It has been accepted for inclusion in Faculty Publications in Food Science and Technology by an authorized administrator of DigitalCommons@University of Nebraska Lincoln. 


\title{
Promises and Prospects of Microbiome Studies
}

\author{
Maria C. Rivera \\ Virginia Commonwealth University \\ mcrivera@vcu.edu \\ Jacques Izard \\ University of Nebraska-Lincoln \\ jizard@unl.edu
}

Since Anthony van Leeuwenhoek, first microscopic observations of the unseen microbiota and the more recent realization that little of the microbes in the biosphere are known, humans have developed a deep curiosity to fully understand the inner workings of the microbial realm. Our ability to characterize the complexity of microbial communities in their natural habitats has dramatically improved over the past decade thanks to advances in high-throughput methodologies. By eliminating the need to isolate and culture individual species, metagenomics approaches have removed many of the obstacles that hindered research in the ecology of mixed microbial consortia, providing valuable information about the diversity, composition, function, and metabolic capability of the community.

Microbes are the unseen majority with the capability to colonize every environment, including our bodies. The establishment and composition of a stable human microbiome is determined by the host

Published (as Chapter 10) in Metagenomics for Microbiology, ed. J. Izard \& M.C. Rivera (Academic Press, 2015), pp 145-159.

http://dx.doi.org/10.1016/B978-0-12-410472-3.00010-5

Copyright (c) 2015 Elsevier Inc. Used by permission.

Published 14 November 2014. 


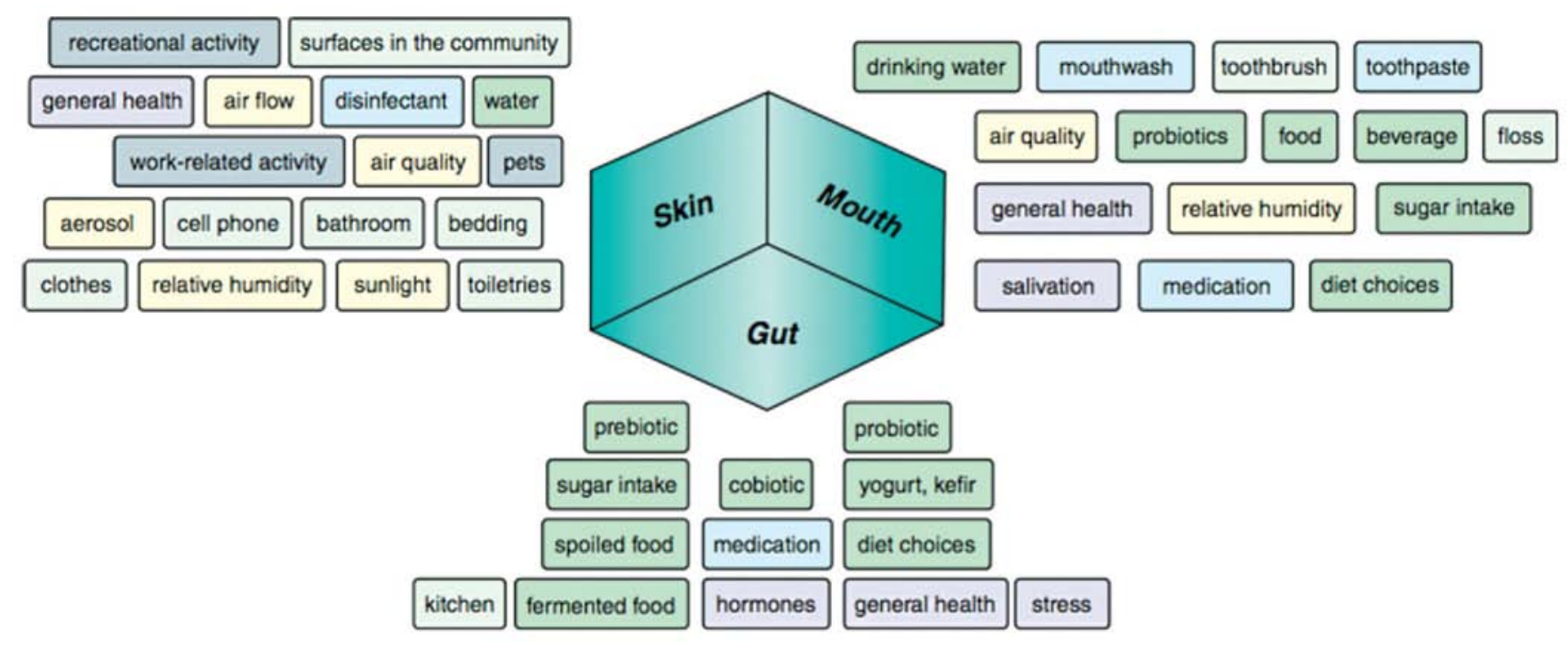

Fig. 1. Microbiome of modern humans is influenced by life-style choices. During everyday life, humans are exposed to many external and internal environmental factors that can influence the composition of their microbiome. The human microbiome is here represented by the microbiota of the skin, mouth, and gut, and next to each body site a series of environmental modifiers. Some of these modifiers, such as general health, diet, medications, and stress, can affect the microbiota of more than one body site, but others, such as toothbrush and toothpaste, can directly modify the microbiota of only one body site.

genetics, immunocompetence, and life-style choices. Our life-style choices determine our exposure to many external and internal environmental factors that permanently or temporarily can influence our microbiome composition. Figure 1 illustrates some of the lifestyle-related factors that might influence the microbiota of the skin, mouth, and gut. It is not limited to what we carry, touch, breath, and eat. Other dispersal vectors include secretion, excretions, aerosols, air flow, animals, moving surfaces, water, beverages, food, contact, wind, tools, toiletry, and others. These influence the microbiome membership, who are present, and they have the ability to participate in the microbiome dynamic within an environment. The establishment of a microbial community is dependent on many environmental factors, including $\mathrm{pH}$, temperature, altitude, weather, soil type, nutrient availability, relative humidity, air quality, pollutants, microbial competitors, and others. In other words, we are superorganisms interconnected with other living forms on this Earth. 
The exploration of the microbial worlds uncovered the extreme microbial diversity throughout the biosphere, from hydrothermal vents on the ocean floor to the intestinal tract of animals. In addition to metagenomics, other technologies including metatranscriptomics, metaproteomics, metabolomics, and metalipidomics can provide better insights of the microbial ecosystems dynamics. Fundamental gaps in knowledge still exist. We will take few examples to discuss the promises of the upcoming discoveries.

\section{Microbiome During Development and Disease}

Since the 1980s, advances in sequencing technologies have uncovered the immense diversity and functional capabilities of the microbial world. More recently, the introduction of next-generation sequencing (NGS) combined with metagenomics approaches has allowed a better understanding of the complexity of the interactions between animals and their associated microbiota, in particular, the gut microbiome.

The digestive tract of animals has coevolved with a diverse and complex microbial community that responds to host diet and provides metabolic signals to the host during its developmental stages among many other functions. ${ }^{1}$ The permeability of the gut facilitates the transport of metabolites produced by the microbiota, which allows the signaling and interactions between the gut microbiota and the host organs and tissues. ${ }^{2}$ Host immune and nervous system as well as behavior including mating can be influenced. ${ }^{1,3,4}$ The use of metagenomics approaches to understand the complex metabolic interactions between the human host and its microbiome has revealed extensive variability in the diversity and composition of the microbiome between individuals and throughout the life-span of the host. A representation of this knowledge is summarized in Figure 2. Our growing knowledge of the magnitude and complexity of the interactions between the host and the gut microbiome is drastically changing our views of human health, disease, and aging. ${ }^{2}$ The lifelong changes in the complexity of the host-microbiome metabolic interactions offer the opportunity for

the development of specific therapeutic interventions targeting the gut microbiome throughout the life-span of the host. 


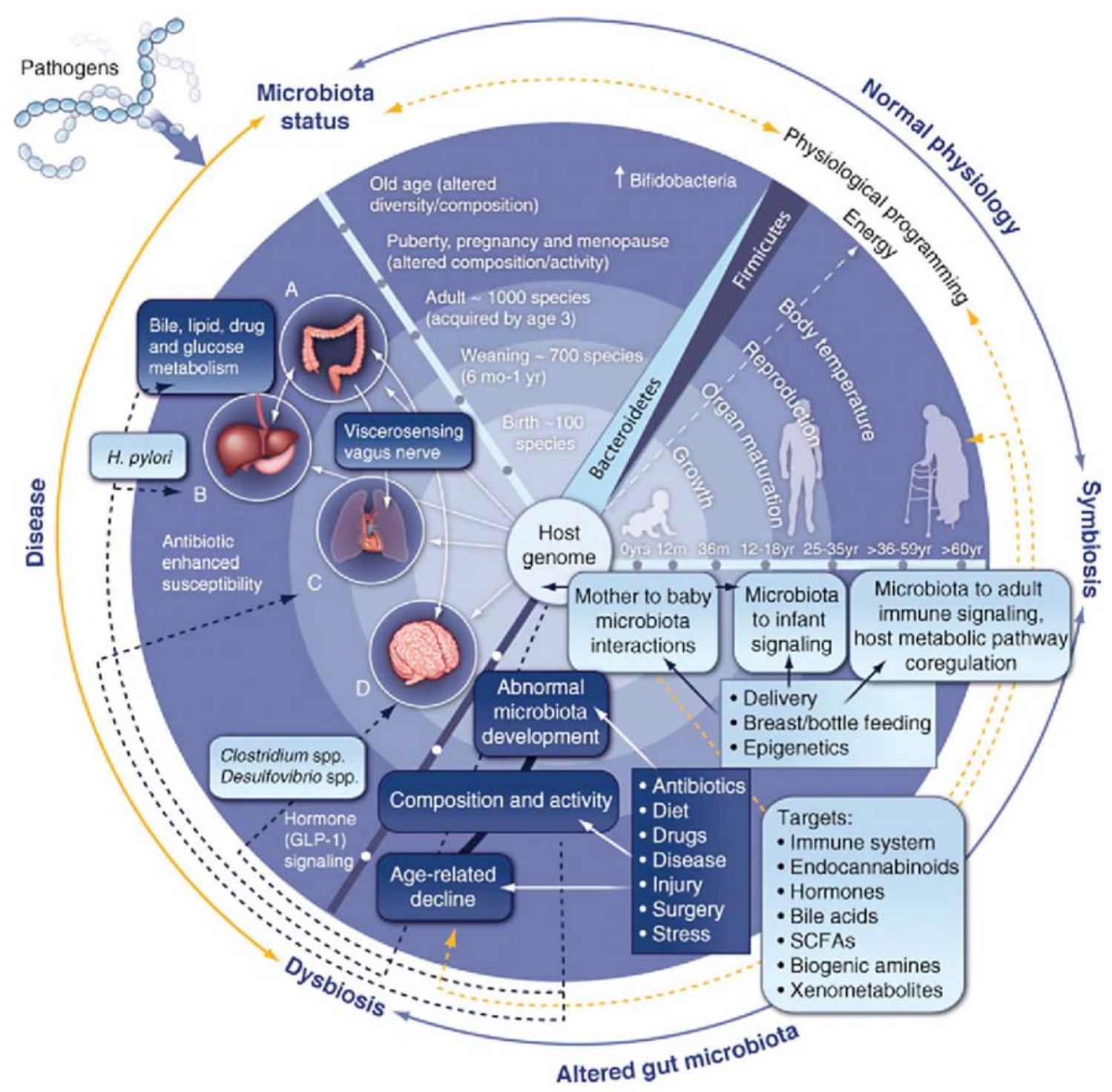

Fig. 2. Gut microbiota in development and disease. The influence of the gut microbiota on human health is continuous from birth to old age. The maternal microbiota may influence both the intrauterine environment and the postnatal health of the fetus. At birth, about 100 microbial species populate the colon. Early environmental factors (e.g., method of delivery), nutritional factors (e.g., breastfeeding or bottle feeding), and epigenetic factors have been implicated in the development of a healthy gut and its microbial symbionts. Changes in gut microbial composition in early life can influence risk for developing disease later in life. During suckling, the microbial community develops rapidly; shifts in microbial diversity occur throughout childhood and adult life, and in old age, there is a decrease in the Bacteroidetes and an increase in the Firmicutes species. The gut microbiota is important for maintaining normal physiology and energy production throughout 


\section{Therapeutic Potential of Microbiota Modifications}

Metagenomic studies have uncovered the connections between the disease and the changes in the homeostasis of host-microbiota interactions. ${ }^{2}$ Such findings have renewed the interest of the medical community toward therapeutic interventions targeting the gut microbiota. Although unknowingly, humans have performed microbial modification for hundreds of years, the first deliberate use of microbial modification for human health date from the beginning of the last century. 5,6 In 1907, Ellie Metchnioff suggested that the consumption of lactic acid bacteria could improve health and longevity, and initiated the modern probiotics movement. Probiotics are "live microorganisms that, when administered in adequate amounts, confer a health benefit on the host." 5,6 Although the application of comparative genomics approaches have contributed to elucidate the genetic components that confer probiotic properties to certain taxa, metagenomics methodology allows more comprehensive studies of the effect of probiotics. ${ }^{5,7}$ Metagenomics together with other omics approaches can potentially identify the metabolites and metabolic pathways inducing the host-microbiota feedback mechanism by which probiotics can modulate health.

Extensive multi-omics studies are needed to fully understand how changes in the ecology of the gut microbiota relate to disease and how to better design and utilize microbial modification therapies. ${ }^{8}$ Which are the microbiome structural and homeostatic changes associated with the development of secondary infections following the

life. Body temperature regulation, reproduction, and tissue growth are energydependent processes that may rely in part on gut microbial energy production. Extrinsic environmental factors (such as antibiotic use, diet, stress, disease, and injury) and the mammalian host genome continually influence the diversity and function of the gut microbiota with implications for human health. Disruption of the gut microbiota (dysbiosis) can lead to a variety of different diseases, including (A) inflammatory bowel disease, colon cancer, and irritable bowel syndrome; (B) gastric ulcers, nonalcoholic fatty liver disease, and obesity and metabolic syndromes; (C) asthma, atopy, and hypertension; and (D) mood and behavior through hormone signaling. The gut microbiota is also important for drug metabolism and preventing the establishment of pathogenic microbes. (Reprinted with permission from the American Association for the Advancement of Science. ${ }^{2}$ ) 
administration of broad-spectrum antimicrobials? ${ }^{9}$ How are the homeostasis and ecological dynamics of the host-microbiome interactions affected by the elimination of specific taxa after immunization? Microbiota modification will soon fall into the realm of personalized and preventive medicine. ${ }^{10-13}$

So far, the best evidence of the therapeutic potential of microbiota modifications is fecal microbiota transplantation (FMT). The replacement of the patient's gut microbiome with the microbiome from a healthy donor has been very successful in the treatment of antibiotic-resistant Clostridium difficile infections. More in-depth metagenomics studies from patients with $C$. difficile infections before and after fecal transplants are needed to determine if it is possible to obtain the same therapeutic benefits by introducing selected members of the microbial community or if indeed the full replacement of the gut microbiome is needed. ${ }^{14,15}$ The efficacy of therapeutic interventions based on microbiota modulation will have to be demonstrated by their ability to consistently restore a healthy steady state. The success of the first randomized clinical trial demonstrating the effectiveness of the FMT for the treatment of recurrent $C$. difficile infections has catalyzed the formation of FMT-patient advocacy groups, and the establishment of stools banks to ultimately encourage the development of targeted therapies. ${ }^{14,16,17}$ The success of FMT suggests that it might be appropriate to establish strict regulations for the screening of infectious disease in microbiome transplants, similar to the ones in place for organ transplants. ${ }^{14,16,17}$

\section{Challenges Ahead: Human Microbiome Information in the Health Care System}

In November 2013, the US Food and Drug Administration (FDA) approved the marketing of the NGS systems as diagnostic devices for human genome sequencing. ${ }^{18,19}$ The authorization by the FDA of the Illumina sequencing platform for whole human genome surveys in the clinical setting paves the way for the development of new sequencing-based clinical tests, potentially including microbiome profiling. It is predicted that in the future, the patient genome and microbiome data can be integrated allowing the identification of medically relevant variants that might transform clinical research and patient care. 
Potentially, these combined data sets can help inform about disease predisposition or responses to drugs allowing the design of personalized care and/or early therapeutic interventions. Many challenges need to be conquered before microbiome information is routinely incorporated in health care.

Extensive studies of microbiome profile variations among healthy individuals are needed before microbiome data can be used for predicting disease predisposition, onset, and progression, and drug-response modulation. Similar to the human genome sequencing, the full integration of microbiome data in the clinical setting requires major research efforts in the collection of rigorous evidence supporting the role of the microbiome in health and disease, the development of appropriate regulatory and validation policies, the implementation of policies addressing patients' rights, and the training of the physician and health care professionals in microbiome data interpretation. ${ }^{20} \mathrm{~A}$ lot of research and validation will be required before the approval by the FDA of microbiome profiling in the clinical setting. Accuracy, precision, analytical sensitivity and specificity, reference range, and reportable range will be scrutinized. In the United States, the Clinical Laboratory Improvement Amendments (CLIA), which perform testing of clinical laboratories to ensure their accuracy in testing human samples, will have to develop new standards that can capture the microbiome complexity. As many other technological advances and discoveries, there is likely to be a lapse of time before the actual adoption of the new technology into the clinical setting.

\section{Ethical Considerations of Microbiome Research}

In the 21st century, the Internet and other digital technologies have facilitated the access to personal information raising concerns related to privacy and data rights issues. The ease of the data accessibility has serious implications for the regulation of research in human subjects. In microbiome research, those regulatory issues are related to the selection and recruitment of human subjects, the possibility of individual or group stigma associated with the research findings, privacy and confidentiality, and informational risks associated with disclosure of some of the findings. ${ }^{21-25}$ Additionally, microbiome research subjects may be identified by the disclosure of information collected 
from the behaviors survey and/or from the microbiota samples. In addition to the issues related to research-generated data, unique to the human microbiome are issues related to informed consent for future use of stored microbiota samples. ${ }^{26-28}$ "Who owns your poop?" is the humorous and thought-provoking question posed by Alice Hawkins and Kieran O'Doherty, while discussing the impact on microbiome research of issues related to privacy, consent, ownership, return of results, governance, and benefit sharing. ${ }^{28}$ Although some ethical issues are unique to microbiome research, similar ethical concerns were extensively debated during the Human Genome Project and, more recently, genome-wide association studies.24,29

To better address the ethical issues facing contemporary research such as microbiome research, in 2011, the US Department of Health and Human Services issued an Advance Notice of Proposed Rulemaking (ANPRM), entitled "Human Subjects Research Protections: Enhancing Protections for Research Subjects and Reducing Burden, Delay, and Ambiguity for Investigators," proposing changes to the "Common Rule," as the current federal policy for the Protection of Human Subjects is known. ${ }^{43}$ Some of the ANPRM proposed changes include: (1) adjusting Institutional Review Board (IRB) review to contemporary research, (2) establishing a single IRB review for multi-institution research, (3) specific written consent for the use of biospecimens, (4) standards for data security and protection of identifiable or potentially identifiable data, (5) implementing a systematic approach to the collection and analysis of data on unanticipated problems and adverse events, and (6) extending the federal rules to apply to all research, regardless of funding source, that is conducted at US institutions that receive some funding from a Common Rule agency for research using human subjects. The proposed revisions to the Common Rule present many challenges to the regulation of research using human subjects deserving immediate attention by the research community. ${ }^{30}$

The ethical issues facing microbiome research require the implementation of novel guidelines on the proper and ethical collection and use of the data generated by this technology. At the same time, to stimulate and facilitate the process of discovery and to keep pace with the latest technological advances, it is imperative for the regulatory agencies to implement a more agile and adaptable evaluation system. 
The artist, like the scientist, can expose and highlight the ethical and privacy issues associated with new technologies. To raise awareness of our increasing access to biotechnologies, Gabriel Barcia-Colombo created the art installation 'DNA vending machine'. ${ }^{31}$ The installation shows visuals of individuals whom donated mouthwash from which DNA was extracted, as well as a packaged vial of that DNA. The installation is both troubling and natural. Both oral microbiome and human DNA should be present in the beautifully condensed genetic information presented by the artist. One can just wonder how such material can be used, and if we should expect prepackaged microbiomes to be on the supermarket shelves.

Another side of this ethics debate is whether the microbiota has rights. Under the assumption "for the benefit of all," we have the technological capacity to permanently eradiate members of the microbiota or, through synthetic biology, "create new microbiota." ${ }^{32-34}$

\section{Citizen Scientist, Crowdsourced Research, and the Microbiome}

The increasing evidence suggesting the important role played by the microbiota in development, aging, and many human diseases has spur great interest from the general public and has introduced the microbiome field to the citizen science movement. ${ }^{35-37}$ Using the citizen science principles of crowdsourcing and crowdfunding, companies and open-sourced projects have been established to collect microbiome samples from donors and provide them with a snapshot of their own microbiome profile in exchange for a monetary contribution. ${ }^{33}$ Some large projects include the American Gut in association with the Human Food Project and several universities, the Home Microbiome study with the Alfred P. Sloan Foundation and Argonne National Laboratories, and the Project MERCCURI to analyze the space station microbiome. Online resources, such as SciStarter and microBEnet, allow the identification of active studies where individuals can be involved. The popularity and success of these types of projects is attributed to the need of the general public to access scientific information that can potentially impact their lives and health. This need of information is strongly felt in the case of the gut microbiome, as changes in the gut microbiota have been associated with several debilitating human conditions. 
The citizen science movement can empower the individuals but at the same time raises several ethical issues and imposes additional responsibilities and concerns to the researchers involved in such projects. ${ }^{35,38-41}$ The research community needs to be aware of the implicit expectations of the citizen scientists and making sure that data collection is not the only goal of the project. While moderating the high expectations of the citizen scientist, the researchers need to ensure the proper use of the collected data to help improve the health and knowledge of, for example, the human subjects. ${ }^{42}$ Properly used and integrated with other health status data, the generated microbiome profiles can potentially provide information very useful to both the human subjects and the research community. If rigorous studies corroborate the observed associations between disease state and changes in microbiome structure, the information could improve preventive health care and lead to earlier medical interventions.

Although of immense benefit to the research community and to the participants, the modality of data collection by citizen scientist presents serious concerns with privacy and security of the data. Microbiome samples contain not only the individuals' microbiome but also the genetic information that could potentially identify the sample donor. A successful microbiome citizen science project requires the implementation of the best practices for collection, management, security, analysis, and communication of all the data collected. ${ }^{21-24}$ It is important to point out the well-known fact that research conducted using data collected from self-selected participants have methodological limitations. Because of selection bias, information bias, and confounding effects, the findings of research using self-selected participants requires cautious interpretation..$^{40}$ It requires the implementation of the proper analytical methodology to identify and possibly compensate for those biases and confounding effects. ${ }^{40}$ This type of collection efforts require the full awareness of the participants that the observed correlation or associations might lack accuracy or generality because of those methodological limitations.

The use of self-selected participants can potentially restrict the health benefits of microbiome profiling to a small sector of the population, because the data collected is skewed based on socioeconomics, ethnicity, and/or disease state of the donor. Given the current funding situation, it can also limit the testing of alternate hypotheses. ${ }^{35-41}$ 
These data-gathering efforts will facilitate the collection of large number of samples and help the democratization of science by empowering the citizen scientist.

\section{Metagenomics, Agriculture, and Food Microbiology}

For living entities, proper nutrition is a key factor for survival. Agriculture and food microbiology can benefit from metagenomics advances by improving food safety and security, improving the detection of threats to food production and supply, and increasing the productivity of domestic animals and plants. In meat production, the ongoing use of direct-fed microbial could also be optimized in function of the food intake and the animal of choice.

The World Health Organization estimates that worldwide, approximately 2.2 million annual deaths are associated with foodborne and waterborne pathogens, including bacteria, viruses, and parasites. ${ }^{43}$ Food and water safety is a worldwide necessity. The depth and high coverage of NGS makes metagenomics a powerful tool in the detection and surveillance of foodborne pathogens, the detection of outbreaks and transmission routes of foodborne diseases, the testing of foods and food-associated environments, and the identification of microbiota that may protect against foodborne illnesses.

Although the use of metagenomics approaches to test foods for the presence of specific foodborne pathogens can be problematic, its application successfully identified the presence of serovars of Salmonella in samples that tested negative by bacteriological analytical manual methods and real-time polymerase chain reaction. ${ }^{43,44}$ In order to facilitate the use of metagenomics by governmental agencies and the food industry, it is crucial to develop bioinformatics tools tailored to the needs of the food microbiology laboratory and to put in place the appropriate legal and ethical framework for the collection and use of the data generated. The use of metagenomics approaches in the food industry, to better understand, the food-associated microbial communities, can lead to improvements in productivity, quality, and safety of food. 43,45

Eliminating hunger worldwide, providing desirable food to a larger population, and producing the needed food in a sustainable way are 
major challenges facing future food security ${ }^{46,47} \mathrm{~A}$ possible strategy for meeting those challenges in a sustainable way is to increase agricultural yields and production limits by manipulating above-belowground plant interactions with the goal of reducing pests and increasing crop growth. The association between plants and belowground soil-borne microbiota increases plant fitness. Plant growth promoting rhizobacteria (PGPR) are able to influence plant growth and increase plant resistance to herbivores and pathogens. ${ }^{47-49}$ Metagenomics approaches to uncover the interactions between plants and belowground microbiota in agricultural systems are essential to tailor and target these interactions for maximum benefit to the crops. More in-depth research in the composition and function of the rhizosphere microbiome is needed in order to better understand and identify the activity of PGPR bacteria in different crops. The knowledge obtained by the analyses of rhizosphere microbiome can be used to design PGPR-based interventions that promote plant growth, nutrition, and defense against pests in agricultural systems. The inoculation of soils with beneficial bacteria such as PGPR could be a sustainable approach to increase productions of crops without the input of chemical fertilizers. Also, soil microbiota manipulation can be potentially used to increase nitrogen fixation and reduce the use of fertilizers and subsequent nitrogenization resulting in economical and ecological benefits. ${ }^{50,51}$

\section{Summary}

In this overview, we have highlighted some of the promises and prospects of the utilization of omics approaches to the study of microbiomes. Clearly, there is still much work ahead before achieving a comprehensive understanding of the complex dynamics and interactions within the human microbiome and other microbial ecosystems. At this time, microbiome research is moving beyond the identification of genes and/or taxa and toward an emphasis on the application of multi-meta-omics technologies with the goal of sorting out the functions and pathways responsible for the multidirectional interactions between the microbiota, the host, and the environment. A comprehensive understanding of these interactions will contribute to the 
design of more effective, preventive, and therapeutic interventions. It creates opportunities for a new vision of health and disease treatment that was not imagined in the 20th century.

Many of the resources and technologies required to fulfil the promise of the microbiome are already available. The development of novel bioinformatics frameworks and analytical techniques are essential in order to more efficiently mine and synthesize the information of the so-called "Big Data" embedded in the present research or soon to be generated.

It is interesting to note the concept of scale involved. From the interaction at the atomic scale of the enzyme and its substrate, driving, in part, the functional assembly of a microbiome, to the dynamic interactions of biomes, we have moved from the nanometer to the meter range and beyond. Let's be ready to stretch our minds a little further and create the tools to better handle the rising concepts.

This methodological and conceptual revolution has provided the magnification lens needed to better understand the unseen world in front of our eyes, the microbial communities inhabiting this planet for over 3.5 billion years. It is a scientific revolution that will soon reach every citizen.

Acknowledgments - We would like to thank Rebecca S. Misra for drawing Figure 1. We apologize for limiting the number of references on a vast topic. The work was supported by the National Institutes of Health, under grant CA166150 (Jacques Izard), and an award from the Thomas F. and Kate Miller Jeffress Memorial Trust (Maria C. Rivera).

\section{References}

1. Kohl KD, Cary TL, Karasov WH, Dearing MD. Restructuring of the amphibian gut microbiota through metamorphosis. Environ Microbiol Rep 2013;5(6):899-903.

2. Nicholson JK, Holmes E, Kinross J, Burcelin R, Gibson G, Jia W, et al. Host-gut microbiota metabolic interactions. Science 2012;336(6086):1262-7.

3. Sharon G, Segal D, Ringo JM, Hefetz A, Zilber-Rosenberg I, Rosenberg E. Commensal bacteria play a role in mating preference of Drosophila melanogaster. Proc Natl Acad Sci USA 2010;107:2005. 
4. Jost $T$, Lacroix $C$, Braegger $C P$, Chassard $C$. New insights in gut microbiota establishment in healthy breast fed neonates. PLoS One 2012;7(8):e44595.

5. Tissier H. Traitement des infections intestinales par la méthode de la flore bactérienne de l'intestin. Crit Rev Soc Biol 1906;(60):359-61.

6. Anukam KC, Reid G. Probiotics: 100 years (1907-2007) after Elie Metchnikoff's Observation. In: Méndez-Vilas A editor. Microbiology Book Series: Communicating Current Research and Educational Topics and Trends in Applied Microbiology. Spain: FORMATEX; 2007. p. 466-74.

7. Veiga P, Gallini CA, Beal C, Michaud M, Delaney ML, DuBois A, et al. Bifidobacterium animalis subsp. lactis fermented milk product reduces inflammation by altering a niche for colitogenic microbes. Proc Natl Acad Sci USA 2010;107(42):18132-7.

8. Sokol H, Pigneur B, Watterlot L, Lakhdari O, Bermudez-Humaran LG, Gratadoux JJ, et al. Faecalibacterium prausnitzii is an anti-inflammatory commensal bacterium identified by gut microbiota analysis of Crohn disease patients. Proc Natl Acad Sci USA 2008;105(43):16731-6.

9. Antonopoulos DA, Huse SM, Morrison HG, Schmidt TM, Sogin ML, Young VB. Reproducible community dynamics of the gastrointestinal microbiota following antibiotic perturbation. Infect Immun 2009;77(6):2367-75.

10. Guyonnet D, Chassany O, Ducrotte P, Picard C, Mouret M, Mercier CH, et al. Effect of a fermented milk containing Bifidobacterium animalis DN-173 010 on the health-related quality of life and symptoms in irritable bowel syndrome in adults in primary care: a multicentre, randomized, double-blind, controlled trial. Aliment Pharmacol Ther 2007;26(3):475-86.

11. Kekkonen RA, Lummela N, Karjalainen H, Latvala S, Tynkkynen S, Jarvenpaa $\mathrm{S}$, et al. Probiotic intervention has strain-specific anti-inflammatory effects in healthy adults. World J Gastroenterol 2008;14(13):2029-36.

12. Lemon KP, Armitage GC, Relman DA, Fischbach MA. Microbiota-targeted therapies: an ecological perspective. Sci Transl Med 2012;4(137):137rv5.

13. Licht TR, Hansen M, Bergstrom A, Poulsen M, Krath BN, Markowski J, et al. Effects of apples and specific apple components on the cecal environment of conventional rats: role of apple pectin. BMC Microbiol 2010;10:13.

14. Cammarota G, laniro G, Gasbarrini A. Fecal microbiota transplantation for the treatment of Clostridium difficile infection: a systematic review. J Clin Gastroenterol 2014;48(8):693-702.

15. Petrof EO, Gloor GB, Vanner SJ, Weese SJ, Carter D, Daigneault MC, et al. Stool substitute transplant therapy for the eradication of Clostridium difficile infection: "RePOOPulating" the gut. Microbiome 2013;1(1):3.

16. Owens C, Broussard E, Surawicz C. Fecal microbiota transplantation and donor standardization. Trends Microbiol 2013;21(9):443-5.

17. Smith MB, Kelly C, Alm EJ. Policy: How to regulate faecal transplants. Nature 2014; 506(7488):290-1.

18. FDA Press Release N. FDA allows marketing of four "next generation" gene sequencing devices, 2013.

19. Collins FS, Hamburg MA. First FDA authorization for next-generation sequencer. N Engl J Med 2013;369(25):2369-71. 
20. Gargis AS, Kalman L, Berry MW, Bick DP, Dimmock DP, Hambuch T, et al. Assuring the quality of next-generation sequencing in clinical laboratory practice. Nat Biotechnol 2012;30(11):1033-6.

21. Wolf SM, Crock BN, Van Ness B, Lawrenz F, Kahn JP, Beskow LM, et al. Managing incidental findings and research results in genomic research involving biobanks and archived data sets. Genet Med 2012;14(4):361-84.

22. Human Microbiome Project Consortium. A framework for human microbiome research. Nature 2012; 486(7402): 215-21.

23. McGuire AL, Colgrove J, Whitney SN, Diaz CM, Bustillos D, Versalovic J. Ethical, legal, and social considerations in conducting the Human Microbiome Project. Genome Res 2008;18(12):1861-4.

24. Hoffmann DE, Fortenberry JD, Ravel J. Are changes to the common rule necessary to address evolving areas of research? A case study focusing on the human microbiome project. J Law Med Ethics 2013;41(2):454-69.

25. Tryka Ka, Hao L, Sturcke A, Jin Y, Wang ZY, Ziyabari L, et al. NCBI's Database of Genotypes and Phenotypes: dbGaP. Nucleic Acids Res 2014;42:D975-9.

26. Slashinski MJ, McCurdy SA, Achenbaum LS, Whitney SN, McGuire AL. "Snakeoil," "quack medicine," and "industrially cultured organisms": biovalue and the commercialization of human microbiome research. BMC Med Ethics 2012;13:28.

27. Master Z, Nelson E, Murdoch B, Caulfield T. Biobanks, consent and claims of consensus. Nat Methods 2012;9(9):885-8.

28. Hawkins AK, O'Doherty KC. "Who owns your poop?": insights regarding the intersection of human microbiome research and the ELSI aspects of biobanking and related studies. BMC Med Genomics 2011;4:72.

29. Lewis $C$, Hill M, Skirton H, Chitty LS. Non-invasive prenatal diagnosis for fetal sex determination: benefits and disadvantages from the service users' perspective. Eur J Human Genet 2012;20:1127-33.

30. Henry LM. Introduction: revising the common rule: prospects and challenges. J Law, Med Ethics 2013;41:386-9.

31. Barcia-Colombo G. DNA Vending Machine. 2014. (Accessed April 26, 2014) http://www.gabebc.com/DNA-Vending-Machine

32. Cockell CS. The Microbial Stages of Humanity. Interdiscip Sci Rev 2011;36:301-13.

33. Riis S. The ultimate technology: the end of technology and the task of nature. Artif Life 2011;19:471-85.

34. Smith HO, Hutchison CA 3rd, Pfannkoch C, Venter JC. Generating a synthetic genome by whole genome assembly: phiX174 bacteriophage from synthetic oligonucleotides. Proc Natl Acad Sci USA 2003;100(26):15440-5.

35. Riesch $\mathrm{H}$, Potter $\mathrm{C}$. Citizen science as seen by scientists: Methodological, epistemological and ethical dimensions. Public Underst Sci 2014;23(1):107-20.

36. Crall AW, Jordan R, Holfelder K, Newman GJ, Graham J, Waller DM. The impacts of an invasive species citizen science training program on participant attitudes, behavior, and science literacy. Public Underst Sci 2013;22(6):745-64. 
37. Freitag A, Pfeffer MJ. Process, not product: investigating recommendations for improving citizen science "success". PLoS One 2013;8(5):e64079.

38. Cooper CB. Is there a weekend bias in clutch-initiation dates from citizen science? Implications for studies of avian breeding phenology. Int J Biometeorol 2013;58(7):1415-9.

39. Kaye J, Curren L, Anderson N, Edwards K, Fullerton SM, Kanellopoulou N, et al. From patients to partners: participant-centric initiatives in biomedical research. Nat Rev Genet 2012;13(5):371-6.

40. Janssens AC, Kraft P. Research conducted using data obtained through online communities: ethical implications of methodological limitations. PLoS Med 2012;9(10):e1001328.

41. McGuire AL, Achenbaum LS, Whitney SN, Slashinski MJ, Versalovic J, Keitel WA, et al. Perspectives on human microbiome research ethics. J Empir Res Hum Res Ethics 2012;7(3):1-14.

42. Berkman B, Hull S, Eckstein L. The Unintended Implications of Blurring the Line between Research and Clinical Care in a Genomic Age. Personal Med 2014;11(3):285-95.

43. Bergholz TM, Moreno Switt Al, Wiedmann M. Omics approaches in food safety: fulfilling the promise? Trends Microbiol 2014;22(5):275-81.

44. Lukjancenko O, Wassenaar TM, Ussery DW. Comparison of 61 sequenced Escherichia coli genomes. Microb Ecol 2010;60(4):708-20.

45. Ercolini D. High-throughput sequencing and metagenomics: moving forward in the culture-independent analysis of food microbial ecology. Appl Environ Microbiol 2013;79:3148-55.

46. Godfray HCJ, Beddington JR, Crute IR, Haddad L, Lawrence D, Muir JF, et al. Food security: the challenge of feeding 9 billion people. Science 2010;327:812-8.

47. Orrell $P$, Bennett AE. How can we exploit above-belowground interactions to assist in addressing the challenges of food security? Front Plant Sci 2013;4:432.

48. Beneduzi A, Ambrosini A, Passaglia LMP. Plant growth-promoting rhizobacteria (PGPR): Their potential as antagonists and biocontrol agents. Genet Mol Biol 2012;35:1044-51.

49. Pangesti N, Pineda A, Pieterse CM, Dicke M, van Loon JJ. Two-way plant mediated interactions between root-associated microbes and insects: from ecology to mechanisms. Front Plant Sci 2013;4:414.

50. Lugtenberg B, Kamilova F. Plant-growth-promoting rhizobacteria. Annu Rev Microbiol 2009;63:541-56.

51. Mapelli F, Marasco R, Rolli E, Barbato M, Cherif H, Guesmi A, et al. Potential for plant growth promotion of rhizobacteria associated with Salicornia growing in Tunisian hypersaline soils. Biomed Res Int 2013;2013:248078. 